\title{
A Little Quantum Help for Cosmic Censorship and a Step Beyond All That
}

\author{
Nikolaos Pappas \\ Division of Theoretical Physics, Department of Physics, University of Ioannina, 45110 Ioannina, Greece \\ Correspondence should be addressed to Nikolaos Pappas; npappas@cc.uoi.gr
}

Received 27 September 2013; Revised 26 November 2013; Accepted 29 November 2013

Academic Editor: Christian Corda

Copyright (C) 2013 Nikolaos Pappas. This is an open access article distributed under the Creative Commons Attribution License, which permits unrestricted use, distribution, and reproduction in any medium, provided the original work is properly cited.

\begin{abstract}
The hypothesis of cosmic censorship $(\mathrm{CCH})$ plays a crucial role in classical general relativity, namely, to ensure that naked singularities would never emerge, since it predicts that whenever a singularity is formed an event horizon would always develop around it as well, to prevent the former from interacting directly with the rest of the Universe. Should this not be so, naked singularities could eventually form, in which case phenomena beyond our understanding and ability to predict could occur, since at the vicinity of the singularity both predictability and determinism break down even at the classical (e.g., nonquantum) level. More than 40 years after it was proposed, the validity of the hypothesis remains an open question. We reconsider $\mathrm{CCH}$ in both its weak and strong versions, concerning point-like singularities, with respect to the provisions of Heisenberg's uncertainty principle. We argue that the shielding of the singularities from observers at infinity by an event horizon is also quantum mechanically favored, but ultimately it seems more appropriate to accept that singularities never actually form in the usual sense; thus no naked singularity danger exists in the first place.
\end{abstract}

\section{Introduction}

Singularities, conceived as spacetime regions, where curvature (as described by scalar invariant quantities like $R_{\mu \nu \rho \sigma} R^{\mu \nu \rho \sigma}$ ) blows up to exceed any possible upper bound, are one of the most problematic notions in Physics. After all, strictly speaking, if the spacetime metric is ill-behaved at a certain point, then the latter should not be considered as part of that spacetime in the first place. Nevertheless, it is this metric that we rely on to try to describe the properties of that point. We overcome this incoherence by considering an augmented spacetime that contains such singular points as ideal boundary points attached to the ordinary, well-behaved manifold. Since the spacetime structure breaks down at singularities while, at the same time, physical laws presuppose space and time to develop and manifest themselves, naked singularities would be sources of lawlessness, absurdity, and uncontrollable information, therefore an anathema for our perception of the Universe. Even worse, Hawking and Penrose have shown that the emergence of singularities is inevitable in a very large class of universe types, where sufficiently reasonable conditions are satisfied (the theorem actually goes as follows: let $M, g_{a b}$ be a time-oriented spacetime satisfying the following conditions. (A) $R_{a b} V^{a} V^{b} \geq 0$ for any nonspace-like $V^{a}$. (B) The timelike and null generic conditions are fulfilled. (C) There is no closed time-like curve. (D) At least one of the following holds: (Da) there exists a compact achronal set without edge; $(\mathrm{Db})$ there exists a trapped surface; (Dc) there is a $p \in M$ such that the expansion of the future directed null geodesics through $p$ becomes negative along each geodesic. Then $M, g_{a b}$ contains at least one incomplete time-like or null geodesic) [1]. Since all of them are redeemed in our Universe too, singularities are expected with certainty to form in the latter as well. In order to deal with these "monsters," Penrose proposed the famous cosmic censorship hypothesis $(\mathrm{CCH})$ [2]. The weak version of the hypothesis ( $w-\mathrm{CCH}$ ) suggests that observers at infinity can never directly see a singularity, the latter being at all times clothed by an absolute event horizon, whereas its strong version (s-CCH) states that an observer cannot have any direct interaction with a singularity at any time or place [3]. Because of cosmic censorship, then, a naked singularity should never occur except, conceivably, for some special configurations, which are not expected to occur in an actual 
astrophysical circumstance (for a thorough and enlightening presentation of the issues concerning singularities and $\mathrm{CCH}$ see $[4-6])$.

It should be noted here that $\mathrm{CCH}$ does not stem from some well-established physical law or mathematical theorem. Rather, it is a convenient hypothesis that, considering the catastrophic impact of the alternative, we gladly accept as (probably) true. Soon after it was proposed, it was declared as one of the most important open questions in classical general relativity [3], whose derivation remains obscure until now (see [7-13] for reviews on the work done sofar). Initially, arguments supporting the idea were based largely on geometry and issues concerning causality, usually expressed in terms of TIFs and TIPs (terminal indecomposable futures/pasts, resp.) [14] (see again [6]). Penrose was able to derive inequalities involving black hole masses and horizon radii [15] in support of his hypothesis, which interestingly enough were shown to hold true in a series of different situations [16-21]. Moreover, CCH was proven to be valid in various specific spacetimes [22-28]. At the same time, sceptics were trying to construct counterexamples in which naked singularities could emerge [29-37]. However, the majority of those examples presupposed very special and idealized conditions to hold (thus least possible to occur in a realistic universe) to hold, so the credibility of $\mathrm{CCH}$ was far from being fatally undermined by them. Soon it was evident that a quantum treatment was necessary. Besides, the problematic way we describe singularities represents much more our lack of understanding their true nature, namely, the laws of quantum gravity that presumably take over when radii of spacetime curvature of the order of Planck length are attained, rather than their actual behavior. It was proposed (and hoped) by many scientists that the inclusion of quantum phenomena in the picture of gravitational collapse would be the answer to all our difficulties to cope with singularities. In fact, quantum mechanics has been proven very successful in resolving many of the counterexample gedankenexperiments in favor of $\mathrm{CCH}$. More specifically it was used to show that it is impossible to overspin or overcharge a maximal Kerr black hole to produce a naked singularity (a procedure first considered in [38]) [39-41]. In this framework, recent results on the correspondence between Hawking radiation and black holes quasinormal modes [42-45] look to be particularly interesting since they stress too the need for a quantum mechanical approach to the black hole properties if we are to gain a deeper understanding of the latter.

Following this line of thinking we try here to engage quantum mechanics in the treatment of point-like singularities lying at a finite distance (as opposed to singularities lying at infinity or thunderbolts). The key idea proposed is to make appeal to Heisenberg's uncertainty principle,

$$
\Delta x \cdot \Delta p \geq 1 \quad \text { (in natural units where } G=c=\hbar=1 \text { ) }
$$

which we consider the most fundamental feature of every natural system, and check the constraints it imposes, concerning the properties of systems that involve singularities.

\section{Weak Censorship Revisited}

Point-like singularities are expected to form because of the unstoppable collapse of matter that occurs when a too large mass is concentrated in a too small volume. The volume of these singularities would effectively tend to zero by definition; thus they should occupy a single point of spacetime. In the case of a naked singularity, an observer at infinity (i.e., at sufficiently large distance away from it so as to be in an asymptotically flat region of spacetime) would in principle be able to determine its position with arbitrarily high accuracy by, for example, direct observation. When we make a measurement with uncertainty $\Delta x \rightarrow 0$ concerning the position of a quantum system, however, the uncertainty principle states that we have to end up with complete lack of knowledge about its momentum (i.e., $\Delta p \rightarrow \infty$ ), therefore about its energy as well. We argue, though, that this is not the case with naked singularities. Since in principle they can be of arbitrarily large mass, one reasonably expects that the actual procedure of determining their position could not change their momentum significantly. Furthermore, even though quantum gravity is necessary to describe the singularity per se, it is legitimate to anticipate that general relativity is sufficiently accurate to describe spacetime at macroscopic distances away from it. Then, it would be possible to "weigh" the singularity by observing potential gravitational lensing effects or through measuring the trajectory, speed, and acceleration of test bodies that get attracted by it and so forth. This way the mass/energy of the singularity would be known with uncertainty at most of the order of the mass itself ( $\Delta M \sim$ $M)$. All these mean that the existence of a naked singularity, apart from all other undesired consequences, would also violate the uncertainty principle. The conundrum gets settled when the provisions of the $\mathrm{w}-\mathrm{CCH}$ are taken into account. The existence of an event horizon of radius $r_{h} \sim M$, which emerges because of the warping of the spacetime continuum by the singularity mass itself and thus exists in every kind of black hole type, means that the actual position of the singularity can be determined with uncertainty at least $\Delta x \sim$ $r_{h}$. Then we get from (1) that $\Delta p \gtrsim 1 / M$ and consequently we find for the singularity energy the inequality $E \gtrsim 1 / M^{3}$, which obviously is perfectly compatible with measuring its mass/energy with $\Delta M \sim M$. In this sense w-CCH not only is necessary to make general relativity self-consistent, but has a strong quantum support as well.

\section{Strong Censorship Revisited}

What about s-CCH then? It is not hard to imagine a situation where a very large and massive system is in question (e.g., the central region of a galaxy); a trapped surface has already formed while observers living on a planet within the trapped region exist and expect quantum mechanics to hold at all times until they crash into the singularity that will develop some time in their future. Even though the soon-to-form singularity would remain unseen by observers at infinity (so $\mathrm{w}_{-} \mathrm{CCH}$ is satisfied), an observer inside the horizon would actually encounter a naked singularity (being at the same time at a significantly large distance away from it). 
All arguments presented in the paragraphs above hold true for this observer too, so a paradox a rises. The s- $\mathrm{CCH}$ is established to resolve the paradox by predicting that an observer would never actually see the singularity, but since it does not provide us with a mechanism capable of deterring this interaction, it looks more like the expression of a hope than a constraint imposed by some physical law. The only way out, then, is to admit that the notion of unstoppable collapse is wrong and, consequently, no point-like singularity is formed at all. Quantum effects should get so enhanced, at Planck scales, that they would manage to counterbalance the gravitational contracting forces to stop the collapse and prevent singularities from forming in the way we consider them to do today (e.g., the confinement of matter in an everdecreasing volume, which means that it would acquire an ever-increasing momentum/energy, according, once again, to the uncertainty principle, so that it would end up behaving like a highly energetic gas whose pressure would constantly grow to counterbalance eventually the contraction, is a plausible mechanism to be explored in a work to come).

This approach, namely, the expectation that no singularity forms eventually, finds good support from a very interesting result by Geroch which crudely goes as follows: when a manifold admits a Cauchy surface (as is the case for the majority of physically reasonable spacetimes), then it also admits a global time function $t$ that increases along every future-oriented time-like curve, which can be chosen so that every $t=$ const. surface is a Cauchy one. However, Cauchy surfaces cannot intersect the singularity and thus there is no time at which the singularity exists [46].

To sum up, revisiting $\mathrm{CCH}$ on the grounds of the uncertainty principle, we arrive at the conclusion that $\mathrm{w}-\mathrm{CCH}$ should hold true. However, since, by itself, it is insufficient to make the overall picture self-consistent, it is needed that s$\mathrm{CCH}$ also applies. Yet the latter in its turn imposes so strict restrictions; that is, as a way out, one quite naturally arrives to admit that singularities never emerge in the usual sense, rendering $\mathrm{CCH}$, in all its versions, unnecessary in the first place.

\section{References}

[1] S. W. Hawking and R. Penrose, "The singularities of gravitational collapse and cosmology," Proceedings of the Royal Society A, vol. 314, no. 1519, pp. 529-548, 1970.

[2] R. Penrose, "Gravitational collapse: the role of general relativity," Revisita del Nuovo Cimento I, vol. 1, pp. 252-276, 1969.

[3] R. Penrose, "Singularities of space-time," in Theoretical Principles in Astrophysics and Relativity, N. R. Lebovitz, W. H. Reid, and P. O. Vandervoort, Eds., pp. 217-243, University of Chicago Press, 1978.

[4] R. Geroch, "What is a singularity in general relativity?" Annals of Physics, vol. 48, no. 3, pp. 526-540, 1968.

[5] J. Earman, Bangs, Crunches, Whimpers and Shrieks, Oxford University Press, 1995.

[6] R. Penrose, "The question of cosmic censorship," in Black Holes and Relativistic Stars, R. Wald, Ed., pp. 103-122, University of Chicago Press, 1998.
[7] R. M. Wald, "Gravitational collapse and cosmic censorship," http://arxiv.org/abs/gr-qc/9710068.

[8] T. P. Singh, "Gravitational collapse, black holes and naked singularities," Journal of Astrophysics \& Astronomy, vol. 20, pp. 221-232, 1999.

[9] W. A. Hiscock, "Magnetic charge, black holes, and cosmic censorship," Annals of Physics, vol. 131, no. 2, pp. 245-268, 1981.

[10] J. D. Bekenstein and C. Rosenzweig, "Stability of the black hole horizon and the Landau ghost," Physical Review D, vol. 50, no. 12, pp. 7239-7143, 1994.

[11] V. E. Hubeny, "Overcharging a black hole and cosmic censorship," Physical Review D, vol. 59, Article ID 064013, 12 pages, 1999.

[12] T. C. Quinn and R. M. Wald, "Energy conservation for point particles undergoing radiation reaction," Physical Review D, vol. 60, no. 6, Article ID 064009, 20 pages, 1999.

[13] S. Hod, "Black-hole polarization and cosmic censorship," Physical Review D, vol. 60, no. 10, Article ID 104031, 3 pages, 1999.

[14] R. Geroch, E. H. Kronheimer, and R. Penrose, "Ideal points in space-time," Proceedings of the Royal Society of London A, vol. 327, pp. 545-567, 1972.

[15] R. Penrose, "Naked Singularities," Annals of the New York Academy of Sciences, vol. 224, pp. 125-134, 1973.

[16] R. Geroch, "Energy extraction," Annals of the New York Academy of Sciences, vol. 224, pp. 108-117, 1973.

[17] G. W. Gibbons, Communications in Mathematical Physics, vol. 27, p. 87, 1972.

[18] T. Willmore and N. J. Hitchin, Global Riemannian Geometry, Ellis Horwood publications, Chichester, UK, 1984.

[19] G. W. Gibbons, Classical and Quantum Gravity, vol. 14, pp. 2905-2915, 1997.

[20] P. S. Jang and R. M. Wald, "The positive energy conjecture and the cosmic censor hypothesis," Journal of Mathematical Physics, vol. 18, no. 1, article 41, 1977.

[21] G. Huisken and T. Ilmanen, “The riemannian penrose inequality," International Mathematics Research Notices, vol. 1997, no. 20, pp. 1045-1058, 1997.

[22] A. Krolak, "Towards the proof of the cosmic censorship hypothesis," Classical and Quantum Gravity, vol. 3, no. 3, pp. 267-280, 1986.

[23] A. Krolak, "Towards the proof of the cosmic censorship hypothesis in cosmological space-times," Journal of Mathematical Physics, vol. 28, no. 1, pp. 138-141, 1987.

[24] A. Krolak, "Strong cosmic censorship and the strong curvature singularities," Journal of Mathematical Physics, vol. 28, no. 11, pp. 2685-2687, 1987.

[25] R. P. A. C. Newman, "A theorem of cosmic censorship: a necessary and sufficient condition for future asymptotic predictability," General Relativity and Gravitation, vol. 16, no. 2, pp. 175-192, 1984.

[26] R. P. A. C. Newman, "Cosmic censorship and conformai transformations," General Relativity and Gravitation, vol. 16, no. 10, pp. 943-953, 1984.

[27] R. P. A. C. Newman, "Cosmic censorship and the strengths of singularities," in Topological Properties and Global Structure of Space-Time, P. G. Bergmann and V. de Sabbata, Eds., pp. 153168, Plenum Press, New York, NY, USA, 1986.

[28] P. T. Chrusciel, J. Isenberg, and V. Moncrief, "Strong cosmic censorship in polarised Gowdy spacetimes," Classical and Quantum Gravity, vol. 1, no. 10, pp. 1671-1680, 1990. 
[29] J. A. Wheeler, in Magic without Magic, J. R. Klauder, Ed., Freeman, San Francisco, Calif, USA, 1972.

[30] P. Yodzis, H. J. Seifert, and H. Muller zum Hagen, "On the occurrence of naked singularities in general relativity," Communications in Mathematical Physics, vol. 34, no. 2, pp. 135-148, 1973.

[31] P. Chrusciel, "On space-times with $U(1) \times U(1)$ symmetric compact Cauchy surfaces," Annals of Physics, vol. 202, no. 1, pp. 100-150, 1990.

[32] S. Shapiro and S. A. Teukolsky, "Formation of naked singularities: the violation of cosmic censorship," Physical Review Letters, vol. 66, no. 8, pp. 994-997, 1991.

[33] M. Choptuik, "Universality and scaling in gravitational collapse of a massless scalar field," Physical Review Letters, vol. 70, no. 1, pp. 9-12, 1993.

[34] D. Christodoulou, "Examples of naked singularity formation in the gravitational collapse of a scalar field," Annals of Mathematics, vol. 140, no. 3, pp. 607-653, 1994.

[35] B. Steinmuller and J. P. Lasota, "Radiating bodies and naked singularities," Physics Letters A, vol. 51, no. 4, pp. 191-192, 1975.

[36] K. Lake and C. Hellaby, "Collapse of radiating fluid spheres," Physical Review D, vol. 24, no. 12, pp. 3019-3022, 1981.

[37] P. S. Joshi, Global Aspects in Gravitation and Cosmology, Clarendon, Oxford, UK, 1993.

[38] R. M. Wald, “On perturbations of a Kerr black hole," Journal of Mathematical Physics, vol. 14, no. 10, pp. 1453-1461, 1973.

[39] S. Hod, "Return of the quantum cosmic censor," Physics Letters $B$, vol. 668, no. 4, pp. 346-349, 2008.

[40] G. E. A. Matsas, M. Richartz, A. Saa, A. R. R. da Silva, and D. A. T. Vanzella, "Can quantum mechanics fool the cosmic censor?" Physical Review D, vol. 79, Article ID 101502, 2009.

[41] S. Hod and T. Piran, "Cosmic censorship: the role of quantum gravity," General Relativity and Gravitation, vol. 32, pp. 23332338, 2000.

[42] C. Corda, S. H. Hendi, R. Katebi, and N. O. Schmidt, "Effective state, Hawking radiation and quasi-normal modes for Kerr black holes," Journal of High Energy Physics, vol. 2013, article 8, 2013.

[43] C. Corda, "Effective temperature, Hawking radiation and quasinormal modes," International Journal of Modern Physics D, vol. 21, no. 11, Article ID 1242023, 2012.

[44] C. Corda, "Effective state, Hawking radiation and quasi-normal modes for Kerr black holes," Journal of High Energy Physics, vol. 2013, article 8, 2013.

[45] C. Corda, "Black hole quantum spectrum," The European Physical Journal C, vol. 73, article 2665, 2013.

[46] R. Geroch, "Domain of dependence," Journal of Mathematical Physics, vol. 11, no. 2, pp. 437-449, 1970. 

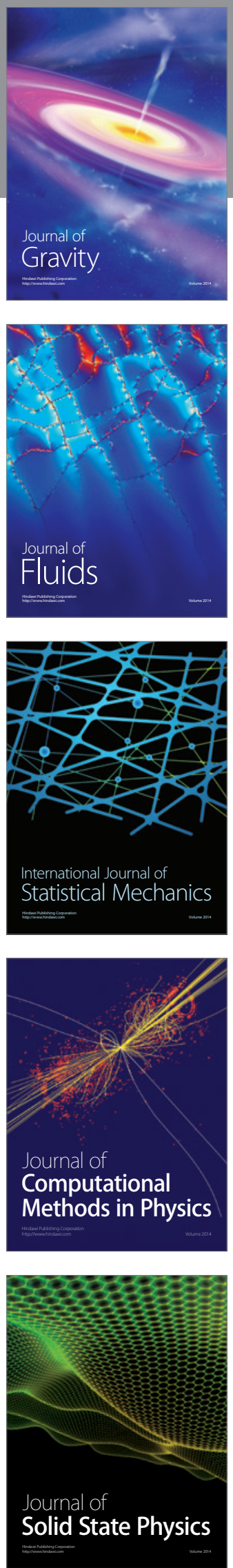

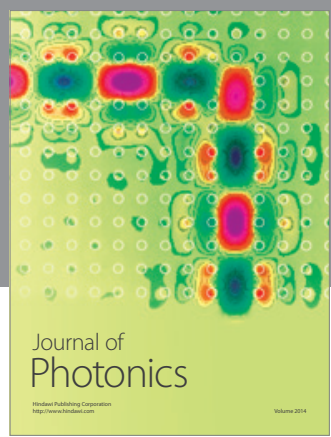

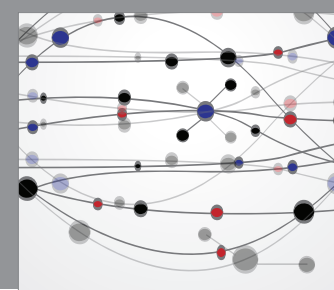

The Scientific World Journal

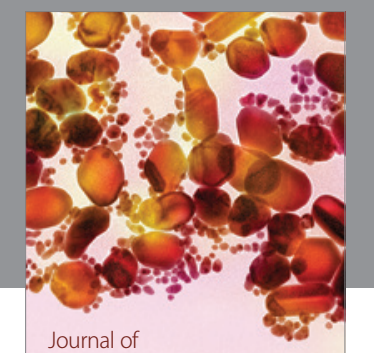

Soft Matter
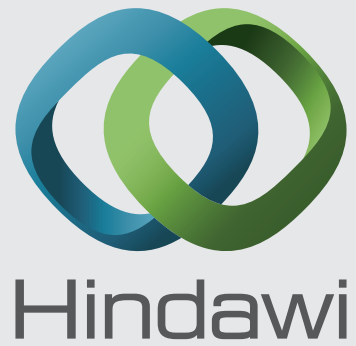

Submit your manuscripts at

http://www.hindawi.com
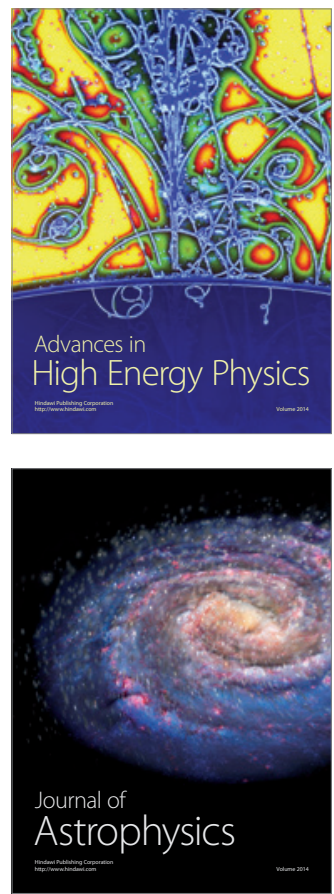
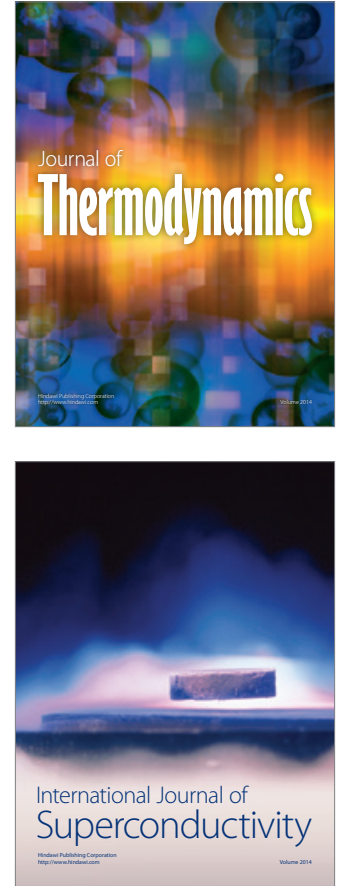
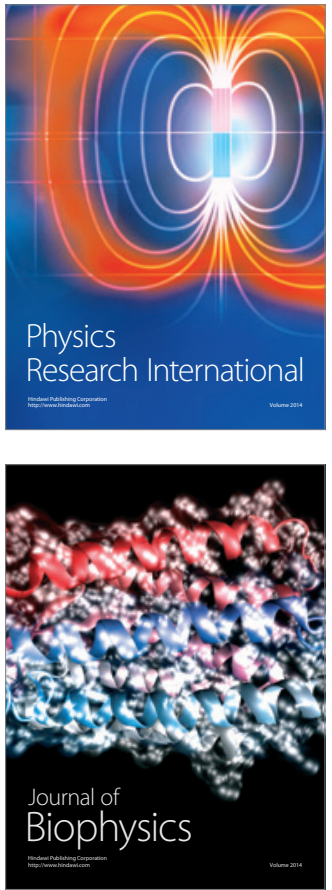
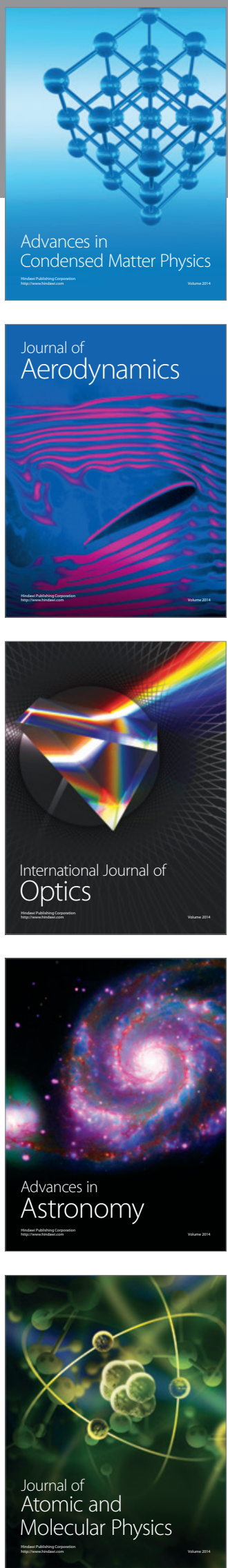